\title{
ELEMENTOS QUE INFLUYEN EN EL FRACASO UNIVERSITARIO: UN ESTUDIO DESCRIPTIVO
}

\author{
INFLUENCING ELEMENTS IN COLLEGE FAILURE: \\ A DESCRIPTIVE STUDY
}

\author{
Pilar Alonso Martín* y Herminia Lobato Soriano** \\ Universidad de Huelva
}

\section{RESUMEN}

El presente trabajo pretende describir algunos de los elementos que intervienen en el fracaso universitario y para ello se construyó un cuestionario que fue cumplimentado de manera anónima por los alumnos que aceptaron participar. El estudio se realizó en los dos curso de la Licenciatura de Psicopedagogía (licenciatura de $2^{\circ}$ ciclo), perteneciente a la Facultad de CC.EE. de la Universidad de Huelva, tanto en el horario de mañana como de tarde, durante los meses de Abril y Mayo del 2002.

Los principales resultados obtenidos muestran que los hábitos de lectura son escasos, quedando reducido a los libros obligatorios de las distintas asignaturas; la media de horas dedicadas al estudio es de 10 horas semanales, tiempo que se reparte en un estudio diario de las asignaturas para la mitad de la muestra. La herramienta más utilizada son los apuntes de clase.

Respecto a los procedimientos y técnicas de estudio se observó que la gran mayoría de la muestra sí las utiliza; el tipo de examen preferido es el combinado (mezcla de tipo test y desarrollo); en cuanto a las dificultades de los dos tipos de examen son el uso de preguntas confusas y la dificultad para estructurar las respuestas.

Además de estas dificultades los tres cuartas partes de la muestra señalan como el principal obstáculo que interfiere en sus estudios la cantidad de trabajos que tienen que realizar para las distintas asignaturas.

Palabras claves: técnicas y procedimientos de estudio, fracaso universitario, tipos de exámenes.

\section{ABSTRACT}

The present work seeks to describe some of the elements that intervene in the university failure and for it was built it a questionnaire that was executed in an anonymous way by the students that accep-

* Es Profesora Asociada en el Departamento de Psicología de la Universidad de Huelva. Líneas de investigación: cursos de doctorado, suficiencia investigadora y en posesión del DEA, realiza su doctorado sobre el test de Bender y su valor discriminativo de problemas emocionales.

** Es Orientadora Laboral en el SOIPEA de la Universidad de Huelva. Líneas de investigación: cursos de doctorado, suficiencia investigadora y en posesión del DEA; realiza su doctorado sobre la violencia entre iguales. Es coautora de varias publicaciones. 
ted to participate. The study was carried out in the Licentiate of Psicopedagogía, belonging to the Ability of CC.EE of the University of Huelva, so much in tomorrow's schedule like of late, during the months of April and May of the 2002.

The main obtained results show that the reading habits are scarce, being reduced to the obligatory books of the different subjects; the stocking of hours dedicated to the study is of 10 weekly hours, time that is distributed in a daily study of the subjects stops half of the sample. The used tool is the class notes.

Regarding the procedures and technical of study it was observed that the great majority of the sample yes it uses them; the type of favorite exam is the cocktail (mixture of type test and development); as for the difficulties of the two exam types they are the use of confused questions and the difficulty to structure the answers.

Besides these difficulties the fourth three parts of the sample point out as the main obstacle that interferes in their studies the quantity of works that you/they have to carry out for the different subjects.

Key words: technical and study procedures, university failure, types of exams.

\section{Introducción}

El fracaso académico entendido como el abandono e los estudios (Saldaña, 1986) o como el importante número de suspensos que en determinadas asignaturas se producen y que conducen, como consecuencia, a un excesivo periodo de actividad académica por encima de lo previsto en los correspondientes planes de estudio, es un problema apremiante al que tiene que enfrentarse la Universidad Española.

Haciendo un breve recorrido por la historia, se puede observar que ya desde el nacimiento de la institución universitaria, las funciones de ésta no diferencian mucho de las actuales, centrándose por un lado, en la transmisión de conocimientos y en la creación del mismo y, por otro lado, en la evaluación del alumno que tenía que demostrar que había aumentado sus conocimientos.

Pero, al mismo ritmo que aumentaba la demanda de la educación superior en nuestro país, a partir de la década de los 60, surge también el aumento de los alumnos que fracasan, aun cuando se asegura que un buen sistema educativo garantiza el acceso a la universidad a grupo de estudiantes que presentan las condiciones, aptitudes y actitudes adecuadas para enfrentarse a las exigencias académicas.

Sin embargo, en los momentos actuales no parece necesario ser un analista riguroso y experimentar con los datos y resultados docentes de nuestras universidades para tomar conciencia del progresivo aumento del fracaso universitario. Por lo que puede entenderse que las respuestas de orientación vocacional y formación humana que se produce desde etapas preuniversitarias no parecen ser suficientes. Por lo cual, se hace necesario investigar el fracaso académico en el ámbito universitario con el fin de identificar los factores de inadaptación entre alumnos e institución.

Esta idea, apoya la demanda que hace De Miguel (1994) de la necesidad de realizar estudios sobre los desajustes entre subsistemas, en los cuales se pretenden explorar la falta de acoplamiento entre la enseñanza universitaria y los tramos educativos anteriores. Este desajuste vendría definido por aspectos como la falta de servicios de orientación o los aspectos didácticos de la enseñanza universitaria, entre otros.

En definitiva, todos los autores (Marín, Infante y Troyano, 2000; Hernández y Pozo, 1996; Martín, Leite, Zurita y Nilda, 2000) establecen que la etiología de los problemas rela- 
cionados con el fracaso escolar de los alumnos universitarios es muy diversa y compleja, que surge de la confluencia de múltiples factores.

De hecho, Marín, Infante y Troyano (2000) recopilan una serie de autores que establecen las siguientes causas:

Según Gylli (1970) podemos hablar de causas fisiológicas, psicológicas y del clima educativo-familiar.

Rodríguez Neira en 1978 distingue entre la falta de voluntad y problemas graves entre los que influyen rasgos patológicos o de desadaptación social.

Tirados (1985) destaca factores tan relevantes como la inteligencia o los aspectos motivacionales y vocacionales.

En esta misma línea Saldaña (1986)constata que los abandonos académicos tienen su origen en la desorientación Vocacional y Gaumann (1971) identifican como factores determinantes del fracaso las aptitudes, las características de la tarea y los determinantes del impulso de activación (motivación, expectativas e intereses).

Como observamos la eficacia del aprendizaje no se relaciona solamente con el potencial cognitivo del estudiante, sino que tiene mucho que ver cómo se utiliza dicho potencial.

González Pineda, 1996 manifiesta que no es de extrañar que haya crecido en los últimos años el interés por estudiar como el alumno utiliza las estrategias de aprendizaje. Definidas por Beltrán (1993) como: "el conjunto de procesos cognitivos encuadrados conjuntamente en un plan de acción, empleados por un sujeto, para abordar con éxito una tarea de aprendizaje".

Son múltiples las clasificaciones que se han establecido acerca de las estrategias de aprendizaje. Pozo (1990) defiende que en la base de todas las clasificaciones existen tres estrategias básicas: estrategias de repaso, de organización y de elaboración.

Danserau (1988) hace una distinción entre lo que él denomina estrategias de aprendizaje propiamente dichas y estrategias de apoyo. Para él las estrategias de aprendizaje son aquellas que actúan directamente sobre el material, y que incluyen desde la comprensión a la utilización, pasando por la retención y la recuperación.

Las estrategias de apoyos son las que hacen referencia a la elaboración y programación de metas y que tratan de mantener un clima cognitivo adecuado para el aprendizaje, fomentando la motivación, la autoestima y la atención.

Según Marín, Infante y Troyano (2000) en el ámbito de la enseñanza universitaria, varios autores (Fullilove y Treisman, 1990; Tait y Entwistle, 1996; Thomas, Bol y Warkentin, 1991) han comprobado que numerosos alumnos universitarios no sólo no están preparados para seguir la enseñanza superior, sino que además tienen gran dificultad para controlar y evaluar sus propias estrategias de aprendizajes.

Por otro lado no debemos olvidar que las estrategias de aprendizaje guardan una gran relación con los estilos de aprendizajes, pudiéndose distinguir tres tipos: el superficial y el profundo, propuestos por Marton, Hounsell y Entwistle en 1984, y el estratégico propuesto por este último autor en 1987.

Según estos autores, un estilo de aprendizaje profundo supone la intención de comprender y el intento de relacionar la información nueva con las experiencias y los conocimientos previos con el fin de extraer significados personales, es decir, hace referencia a lo que en el ámbito educativo conocemos como aprendizaje significativo. 
Por su parte, en un estilo de aprendizaje superficial, la intención del alumno consiste únicamente en satisfacer los requisitos de la tares. Mientras que en el estilo estratégico, aunque el alumno también esta centrado en la evaluación, lo hace con la intención de obtener los mejores resultados.

Centrándonos en el enfoque Psicopedagógico consideramos que la investigación debe centrarse en el proceso de enseñanza-aprendizaje que se desarrolla en la Universidad. Pues los docentes deben estar preparados para dar a los estudiantes los recursos necesarios para conseguir los objetivos educativos. Planteándonos que la falta de herramientas y una mala organización en el proceso de aprendizaje puedan ser causas destacadas en el fenómeno que aquí tratamos.

Por todo ello, el primer paso a dar es la identificación de las variables que afectan al rendimiento académico, lo que permitirá aplicar medidas preventivas que reduzcan la probabilidad de experimentar fracaso en la universidad y por eso el objetivo de nuestro estudio va a centrarse en la descripción de qué herramientas utilizan y cuáles no para enfrentarse al aprendizaje de las distintas asignaturas en cursos de segundo ciclo y determinar si existe una necesidad de plantear unos cursos sobre técnicas de estudio o de herramientas cognitivas para reducir el fracaso de los universitarios.

\section{Métodología}

\section{Muestra}

La muestra del presente trabajo está constituida por 180 sujetos, pertenecientes a los dos cursos que forman parte de la Licenciatura de Psicopedagogía, de ambos sexos $(19 \%$ de hombre y $79 \%$ de mujeres) (Fig. 1) residentes en la ciudad de Huelva, con un rango de edad de 22 a 24 años.

El sistema de muestreo utilizado fue aleatorio por conglomerado.

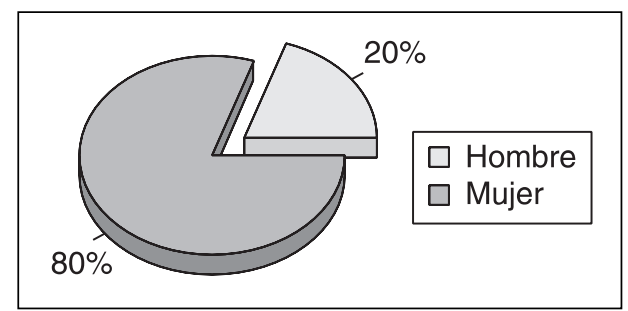

FIGURA 1.

Distribución de sexos.

\section{Instrumento y variables}

Para el estudio se construyó un cuestionario ${ }^{1}$ que consta de 33 ítems, agrupados en diversas áreas temáticas. Se trata de un cuestionario anónimo que se compone de ítems simi-

\footnotetext{
${ }^{1}$ Ver Anexo.
} 
lares a los de otro estudio realizado en nuestro país en el ámbito de la Psicología (Martín Cabrera, E., García García, L. y Hernández Hernández, P., 1999). Esta similitud del cuestionario responde a una revisión realizada, previa a su construcción, de los diferentes instrumentos utilizados en tales estudios, con el objetivo de utilizar ítems que pudiesen facilitar la comprobación de los resultados.

Las variables analizadas son las siguientes:

- Datos sociobiográficos: estado civil, procedencia, situación laboral, disfrute de beca.

- Hábitos de lectura: número de libros que leen o consultan para las asignaturas y para su aprendizaje.

- Hábitos de estudio: Tiempo de estudio: diario, semanal y mensual

Herramientas que utilizan para estudiar

Técnicas de estudio que emplean.

- Dificultades de estudio: Tipos de examen

Dificultades emocionales y de acomodación

Dificultades de aprendizaje.

\section{Procedimiento}

La recogida de información se llevo a cabo a través del cuestionario, que fue cumplimentado de forma anónima, por los alumnos de $4^{\circ}$ de psicopedagogía.

El estudio se realizó en la Facultad de CC.EE., en horario de mañana y tarde durante los meses de abril y mayo de 2002 .

El tiempo empleado en cumplimentar el cuestionario fue entre 20 y 30 minutos, no presentando problemas de comprensión.

El índice alfa de Combranch fue de 0,79.

\section{Análisis de datos}

Para alcanzar los objetivos mencionados se han realizado análisis univariables y bivariables, tales como análisis de frecuencia y pruebas de chi-cuadrado.

El valor de significación se ha establecido para $\mathrm{p}<0.05$

\section{Resultados}

\section{Hábitos de lectura (Tabla 1)}

El 2,8\% de los alumnos afirma leer menos de dos libros no obligatorios, mientras que el $22 \%$ leen entre 2 y 4 libros, siendo el 3,5\% los que leen más de 4 libros al cuatrimestre. 
TABLA 1: Distribución de frecuencia en libros Obligatorios y no obligatorios de la carrera.

\begin{tabular}{|l|c|c|}
\hline \multicolumn{3}{|c|}{ LIBROS NO OBLIGATORIOS } \\
\hline & Frecuencia & Porcentaje \\
\hline menos de 2 & 126 & 72 \\
\hline entre 2-4 & 38 & 22 \\
\hline más de 4 & 6 & 6 \\
\hline \multicolumn{3}{|c|}{ LIBROS OBLIGATORIOS } \\
\hline menos de 5 & 115 & 66 \\
\hline entre 5-10 & 48 & 28 \\
\hline más de 10 & 7 & 6 \\
\hline
\end{tabular}

Respecto a los libros obligatorios oscilan en una media de menos de 5 libros según afirma el $66,5 \%$, frente al 4\% que señalan más de 10 libros obligatorios al trimestre.

El 50\% de los alumnos complementan la información de las asignaturas con otros textos.

El género literario más leído es la novela con un 64\%, mientras que las revistas educativas sólo un 16\% señalan que las utilizan, frente al $31 \%$ que afirman no leer ningún tipo de revista.

En general, el $54 \%$ de los sujetos manifiestan que sus padres no fomentan la lectura en casa.

\section{Hábitos de estudio}

Como puede verse en la figura 2, el tiempo medio dedicado al estudio es de 10 horas semanales para un $40 \%$, seguido de 10 a 20 para un $33 \%$ y más de 20 horas para el $25 \%$.

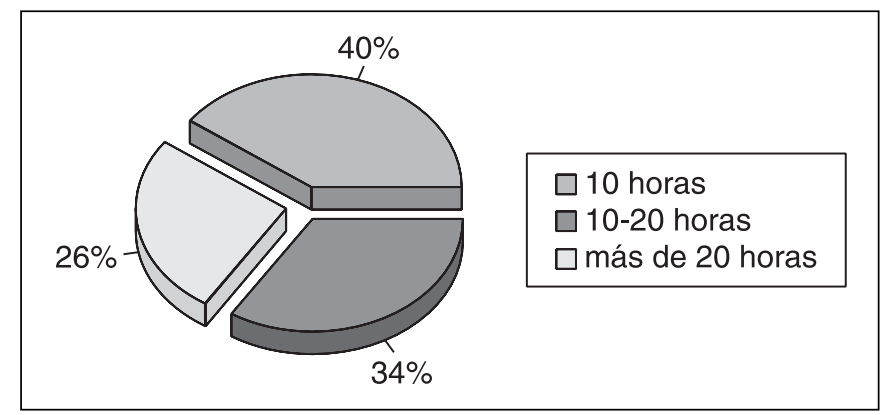

FIGURA 2.

Distribución del tiempo de estudio.

El $50 \%$ de la muestra afirma estudiar diariamente frente al 38\% que sólo estudia en épocas de exámenes (Fig. 3). 


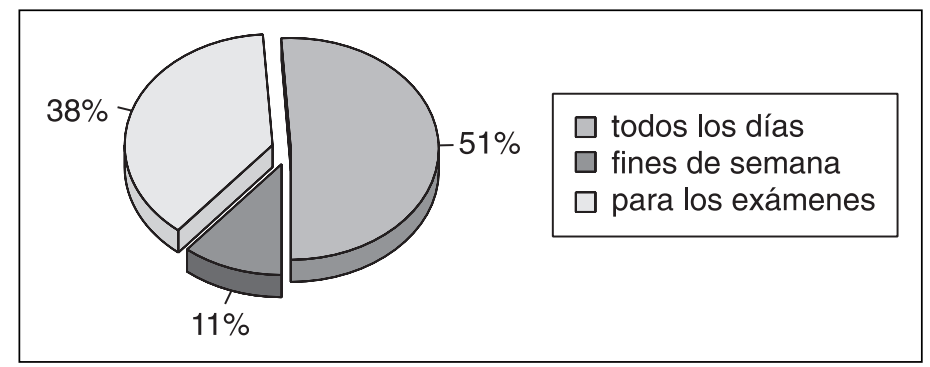

FIGURA 3.

Distribución del momento de estudio.

\section{Herramientas de estudio (Fig. 4)}

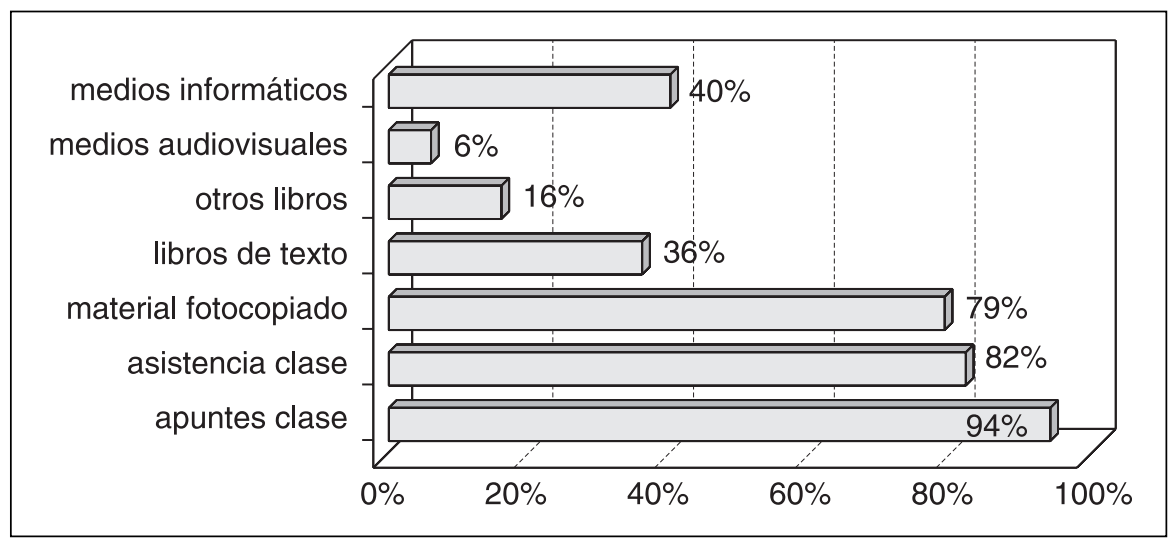

FIGURA 4.

Herramientas de estudio.

Se utiliza tanto los apuntes de clase y las actividades realizadas en las clases obligatorias con un $94 \%$ y un $82 \%$ respectivamente. También se hace uso de material fotocopiado para un $76 \%$, pero sólo el $58 \%$ y el $72 \%$ a veces usan los libros de textos u otros de consulta.

Las herramientas menos usadas son los medios audiovisuales y los medios informáticos que se usan sólo a veces un $50 \%$.

\section{Procedimientos de estudio (Fig. 5)}

Los procedimientos utilizados son el señalar ideas con un $89 \%$, los trabajos de clase con un $83 \%$, relacionar ideas con un $77 \%$, realizar esquemas con un $68 \%$ y resúmenes con un $66 \%$. 


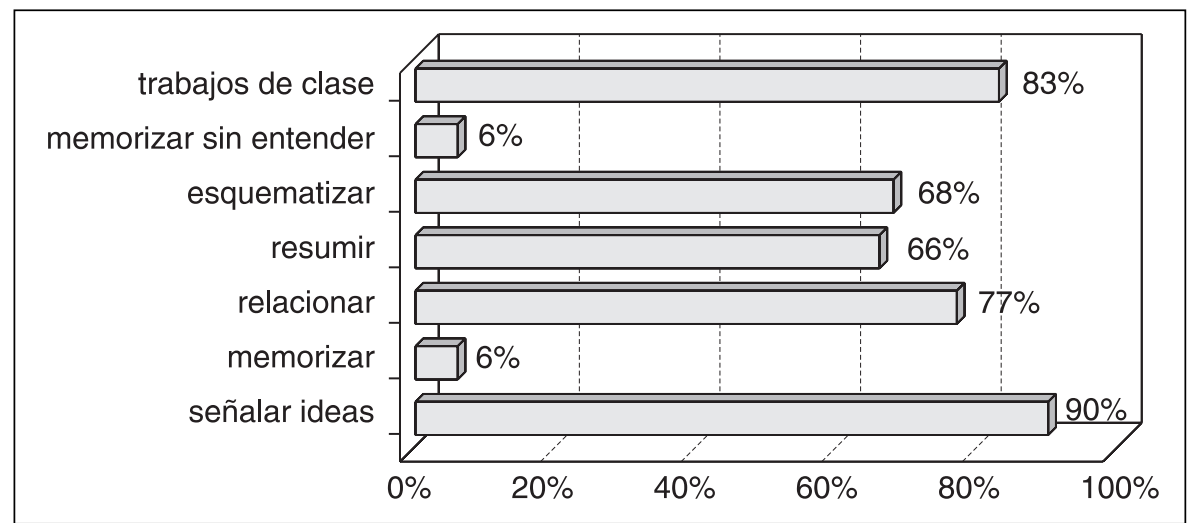

FIGURA 5.

Procedimientos para el estudio.

\section{Tipos de exámenes}

Como se ve en la figuras 6, para el $83 \%$ el tipo de examen preferido es el combinado, entendiéndose por el examen que presenta un parte de preguntas tipo test y otra parte con una o dos preguntas a desarrollar.

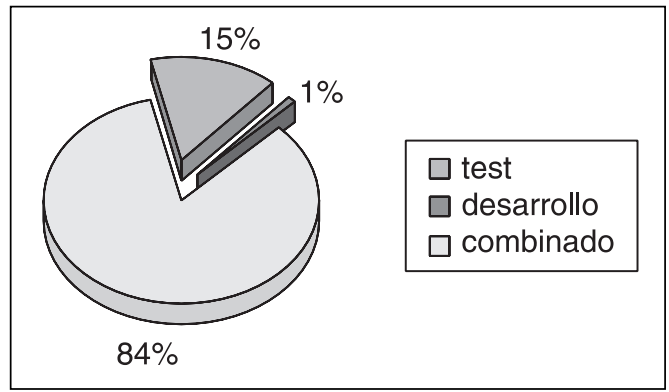

FIGURA 6.

Preferencia de examen.

El motivo de estas preferencias, es como se ve en la tabla 2, por ser más fácil (22\%), permitirles expresar lo que han estudiado y sobre todo porque es donde mejores resultados obtienen $(67 \%)$.

\section{Dificultades para el estudio}

Este bloque se agrupa en torno a tres ejes: el primero referido a las dificultades del examen tipo test, el segundo en las dificultades en el examen de desarrollo y el tercero en una serie de obstáculos que pueden interferir en sus estudios. 
TABLA 2: Motivos de preferencia de examen.

\begin{tabular}{|l|c|}
\hline fácil de recordar & $17 \%$ \\
\hline más objetivo & $9 \%$ \\
\hline más fácil & $22 \%$ \\
\hline muestra lo que sabes & $17 \%$ \\
\hline aprendizaje global & $13 \%$ \\
\hline
\end{tabular}

En la figura 7 puede verse que la dificultad principal en el examen tipo test es la redacción confusa de las preguntas.

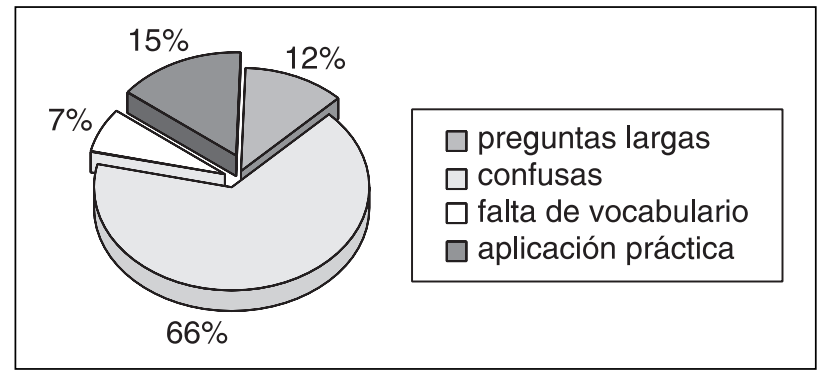

FIGURA 7.

Dificultades del examen tipo test.

En la figura 8 la dificultad de los alumnos se encuantra en la falta de planificación y el modo de estudio este tipo de exámenes.

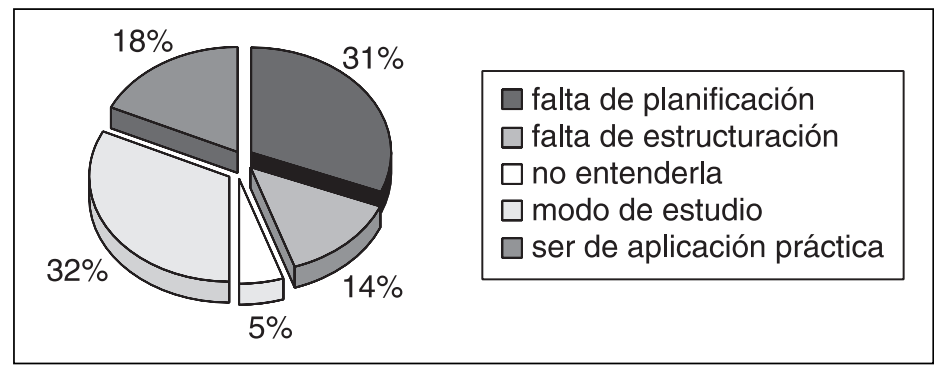

FIGURA 8.

Dificultades del examen tipo desarrollo.

En el tercer bloque observamos que la dificultad principal que señala el $80 \%$ de la muestra es el tiempo invertido en la realización de los numerosos trabajos obligatorios pedidos por los distintos profesores. Dentro de este mismo bloque se destaca la ansiedad ante 
los exámenes para un $20 \%$, mientras que la falte de capacidad es señalada sólo por un $2 \%$ de la muestra.

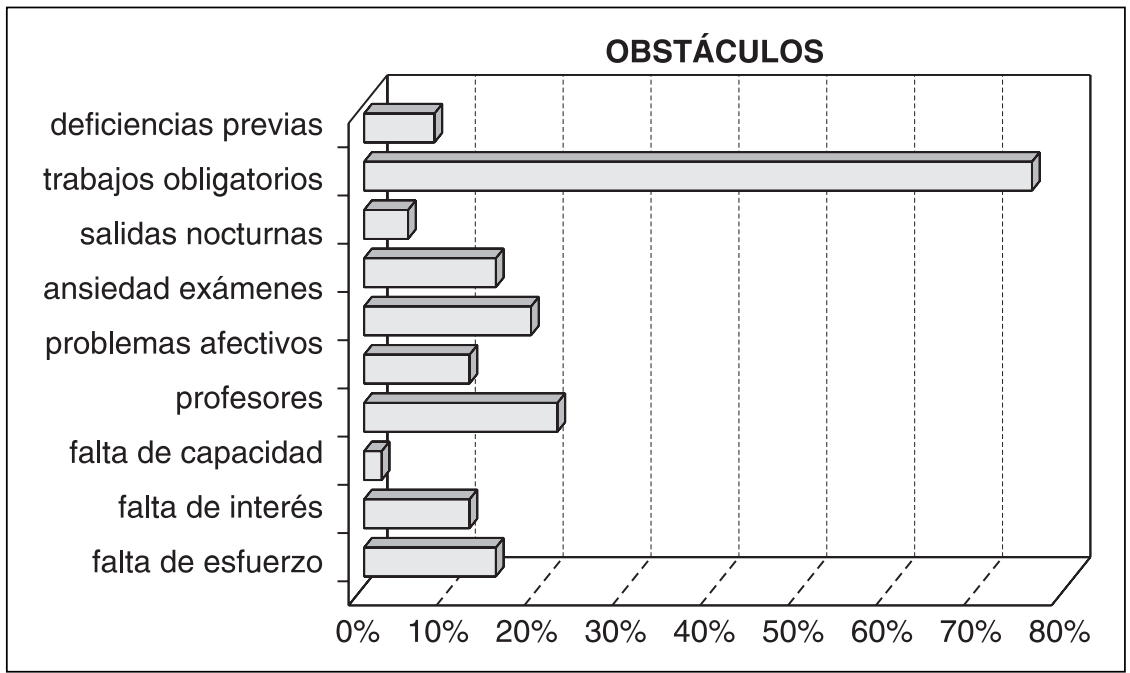

FIGURA 9.

Obstáculos para el estudio.

TABLA 3: Estructuración del material.

\begin{tabular}{|l|c|c|}
\hline Estructuración del material & SI & NO \\
\hline Coherencia & $61 \%$ & $49 \%$ \\
\hline Organizar el material & $83 \%$ & $17 \%$ \\
\hline
\end{tabular}

\section{Discusión}

Dentro de las variables comportamentales encontramos en nuestra muestra una baja actividad lectora, siendo frecuente en el $73 \%$ una lectura de menos de 2 libros anuales que tenga carácter obligatorio y un 31\% que no leen ningún tipo de revista, frente a un $63 \%$ que hacen una lectura del género literario de novela.

Respecto a la temporalización del estudio encontramos, al igual que Samuel Fernández (1989) que el estudiar todos los días de la semana parece imprescindible para tener éxito, llegando a una media de 10 horas semanales de estudio, en comparación con lo encontrado por Martín Cabrera et al (1999) donde la media de las horas semanales ascendió a 18 horas.

En cuanto a estrategias y procedimientos los alumnos sí ven la importancia de señalar ideas, relacionarlas y elaborar resúmenes y esquemas, resultado que se corrobora en el estudio relacionado por Fernández Fernández et al (1991) donde obtiene que los alumnos consideran importantes sintetizar las ideas principales en la toma de apuntes, hacer esquemas, su- 
brayas y tonar notas, así como la comprensión de las explicaciones de clase y estudiar más de lo que se considera necesario para aprobar, mientras que en el estudio de Martín Cabreras (1983) han comprobado que un gran número de estudiantes universitarios, no sólo no están preparados para seguir la enseñanza superior, sino que además tienen dificultades para planificar y evaluar sus propias estrategias de aprendizaje. Como dato curioso en el estudio de Liette y zurita (2000) se observa que los estudiantes piensan que los profesores valoran más que en el examen muestran que han alcanzado un grado de madurez que comprensión de la asignatura, sin embargo en nuestra muestra destacó como procedimiento para el proceso de Enseñanza-aprendizaje con un $40 \%$ el memorizar al pie de la letra y un $31 \%$ el memorizar el contenido sin haberlo comprendido.

Tradicionalmente la inteligencia y las aptitudes mentales han sido las variables que más se han utilizado para intentar predecir el aprendizaje y el rendimiento (González-Pienda, 1996).

En los estudios reseñados anteriormente coinciden en la existencia de una correlación positiva entre capacidad intelectual y resultados exitoso obtenido mientras que en nuestra muestra, los sujetos manifiestan en un $66 \%$ que la capacidad no influye nada en dicho éxito académico.

Respecto al tema de los obstáculos reseñar que el $80 \%$ manifiesta que el mayor porcentaje de horas se las debe dedicar a la realización de trabajos obligatorios, quedando relegado un periodo mínimo de tiempo para la preparación de los exámenes. Por otra parte, la muestra manifestó que su relación con el profesor puede repercutir en un $65 \%$ algo y en un $22 \%$ mucho en el conjunto de actividades académicas, resultado que coincide con Marín (1983) que establece en un 65\% de su muestra, un escaso contacto con los profesores, pudiendo esta relación ser relegada a un nivel académico formal concretizado en consultas e intervenciones en clase.

Un 44\% señala que los problemas afectivos interfieren en sus estudios, de hecho Marín Sánchez (2000) establece que a nivel paraacadémico los cambios en los sentimientos y emociones que pueden afectar al equilibrio personal pueden repercutir negativamente en los estudios.

Un $62 \%$ reseña que no es un obstáculo es la falta de capacidad, en contraste con lo obtenido por Fernández et al. (op. cit.), donde aparece que la capacidad intelectual es, en general, considerada esencial o al menos, importante en todos los estudios, destacando la capacidad de reflexión, el pensamiento lógico y la capacidad de abstracción.

En el tema de la ansiedad, un $75 \%$ declara que es un obstáculo en mayor o menor medida a la hora de conseguir el éxito académico, al igual que manifiesta autores como Marín (1983) y Marie y col. (2000), que indican que la mayoría de los estudiantes universitarios tienen a responder negativamente ante situaciones estresantes y/o que requieren una acción rápida y dinámica y constatando con otros estudios (Peiro y Prieto, 1996) donde añaden que el equilibrio emocional es una variable muy influyente en el rendimiento humano.

\section{Conclusiones}

Las conclusiones más significativas del estudio hacen referencia a la existencia de una serie de variables que sistemáticamente aparecen como relevantes en relación con un mejor 
rendimiento de los universitarios. Por un lado, variables puramente comportamentales, como los hábitos de estudio (estrategias, herramientas...) y por otro lado variables afectivas (ansiedad, problemas afectivos...); así se detecta en nuestra investigación una gran falta de hábitos de lectura, tanto de manuales básicos, como libros que no sean obligatorios que complementen sus conocimientos, o revistas especializadas, donde pueden encontrar las últimas investigaciones. Igualmente se observa una gran dificultad para planificar el tiempo de estudio, concentrándose éste en las épocas de exámenes y por último llama la atención la separación que hace el alumno del tiempo de preparación de trabajos de una asignatura y la preparación del examen de esa misma asignatura, como si el tiempo de trabajo no fuera tiempo de estudio, sino que es vivido como tiempo distinto.

En definitiva, consideramos que a medida que el alumno avanza en el nivel de estudios la influencia de la inteligencia y aptitudes mentales disminuyen como variables predictoras del éxito universitario. Una posible explicación sería que los estudiantes que poseen unas buenas aptitudes académicas son otras variables las que predicen mejor el rendimiento, como pueden ser las variables de apoyo: la falta de planificación del tiempo de estudio, ansiedad ante los exámenes, aprendizaje superficial y la no cumplimentación de los requisitos de la tarea.

Estos resultados dan cuenta de la complejidad del fenómeno estudiado, en el que tiene que ver con la multiplicidad de variables implicadas en el mismo, tanto las más académicas (rendimiento académico, capacidad intelectual...) como las psicológicas (ansiedad, problemas emocionales...).

Esto nos lleva a plantearnos la importancia de una detección temprana del fracaso académico universitario, y si es posible dicha detección también es posible prevenir su aparición mediante estrategias que incidan en las variables antes reseñadas, pudiendo así plantear las intervenciones necesarias para paliar dicho fracaso.

Dicha intervención debería tener en cuenta los aspectos psicopedagógicos estableciéndoles un sistema de control de autorización u orientación valorándose más el trabajo personal realizado por el alumno a lo largo del curso académico, y no solamente la calificación obtenida en el examen final (idea que se encuentra apoyada por la Declaración de Bolonia, 1999).

\section{Referencias bibliográficas}

Albee, G. W. (1980). "A competency Model to replace the Defect Model”. En M. Gibbs, J. R. Lachemmeyer y J. Sigar (Comps.), Community Psychology. New York: Gardner Press.

Baquero, R. et al. (1996). "El alumno Ingresante en la Universidad. Un abordaje psico-educativos". Espacios en blanco. Revista de educación, $\mathrm{n}^{\circ}$ 3-4 junio.

Brenca, R. (1996). Cuando la oralidad y la escritura entran en conflicto. Ponencia al III Congreso Internacional Latino-Americano de Semiótica, IV Congreso Brasileño de Semiótica, Pontificia Universidad Católica de San Pablo, Brasil, 1996.

Carvallo, S. (1997). El discurso caótico: aportes para el análisis crítico. Ponencia al II Coloquio de ALED, Universidad de Buenos Aires, 1997.

Casanova, J., Sanjose, R. y Pérez, P. J. (1985): “Contribución al análisis del fracaso escolar en el primer curso de las Facultades de Ciencias". Revista de Educación, 278: 99-117. 
Casco, M. (1998). Los problemas del léxico en las producciones escritas de estudiantes universitarios. Ponencia al VII Congreso Nacional de la SAL, Neuquén.

Casullo, M. (1996). Evaluación psicológica en el campo socioeducativo. Buenos Aires: Paidós.

Clemente Carrión, A. (1998). "Modificación y rendimiento académico en los estudios superiores". En M. Acosta, (Dir.), Creatividad, motivación y rendimiento académico (pp. 81-97). Granada: Aljibe.

Coulon, A. (1995). Etnometodología y Educación. Buenos Aires: Paidós.

Crespo, J. y Muñoz, A. (1976): “Actitudes y conocimientos sobre los estudios universitarios en alumnos de COU". Revista de Psicología General y Aplicada, 141-142, 1052-1058.

De Beaugrande, R. y Dressler, W. (1981). Introducción a la lingüística del texto. Tübingen: Niemeyer.

Eccles, I. (1983). "Expectancies, values, andacademic behaviors”. En J. T. Spence (Ed.), Achievement and achievement motives (pp. 12-23). San Francisco: Freeman.

Echebarría, A. (comp. 1991). Psicología social socio-cognitiva. Bilbao: Descleé de Brower S.A.

Eco, U. (1989). Lector in fábula. Barcelona: Lumen.

Eysenck, H. J. (1975). "Review of Wankowski and Cox". Educational Review, Vol. 27, n 2.

Feldman, R. S. (1986). Social psychology of education. Cambridge: Cambridge University Press.

González Tirados, M. (1985): Influencia de la naturaleza de los estudios universitario en los estilos de aprendizaje de los sujetos. Tesis doctoral 1985, Universidad Complutense de Madrid.

González Tirados, R. M. (1985). Análisis de las causas del fracaso escolar en la Universidad Politécnica de Madrid. Madrid: CIDE.

González, M. A. y López, E. (1985): "Factores del rendimiento universitario". Revista Española de Pedagogía, XL1II, 169-170: 497-510.

Graumann, C. F. (1971). Fundamentos de psicología. Vol. I. Motivación, Madrid: Morata.

Gylli, M. (1978). El problema del rendimiento escolar. Barcelona: Oikos-Tau.

Herrero, S. R. y Ingestos, A. (1980). El rendimiento académico en la Universidad. Salamanca: ICE

Holder, R. L. y Wankowski, J. A. (1980). Personality and academic performance of students at University. Research into Higher Education Monographs. The Society for Research into Higher Education, University of Surrey, Guilford. Surrev.

Holland, J. L. (1966). The psychology of vocational choices. A theory of personality types and model environments. Braisdell: Waltham.

Holland, J. L. (1985). Making vocational choices. A theory of personality types and model environments. Englewoods Cliffs: Prentice-Hall.

Huertas, I. A. (1997). Motivación. Querer Aprender. Buenos Aires: Paidós.

Juan Espinosa, M., Colom, B. R. y Quiroga, M. A. (1996). La práctica de la psicología diferencial en industria y organizaciones. Madrid: Pirámide.

Kaplan, C. (1997). La Inteligencia Escolarizada. Buenos. Aires: Miño y Dávila.

Kelley, H. H. (1967). “Attribution theory in social psychology”. En D. Levine (Ed.), Nebraska symposium on motivation, Vol. 15, pp. 192-241. Lincoln: University ofNebraska Press.

Latiesa, M. (Comp.) (1986). Estudio longitudinal de una cohorte de alumnos de la Universidad Autónoma de Madrid. Análisis de la deserción universitaria. Madrid: Universidad Autónoma de Madrid.

Leite, A. (1997). Informe final de investigación, Ingreso y desgranamiento Universitario. Una problemática a resolver. Secretaría General de Ciencia y Técnica.

Lofquist, L. H. y Dawis, R. V. (1991). Essentials of Person-environment correspondence counseling. University of Minnesota: University of Minnesota Press. 
McClelland, D. C. (1961). Human motivation. Clenville: Scott Foresman.

Monereo, C. (1994). Estrategias de Enseñanza y Aprendizaje. Madrid: Graó:

Moscovici, S. (1986). Psicología Social II. Buenos.Aires: Paidós.

Morris, L. W., Davis, M. y Hutchins, C. (1981). "Cognitive and emotional components of anxiety". Joumal of Educational Psychology, 73, 541-555.

Núñez Pérez, I. C. (1996). "Motivación en el Ámbito Universitario: concepto de inteligencia, metas de estudio, elección de tarea y aproximaciones al Aprendizaje, Universidad de Oviedo, España". Revista de Educación, n 310 , mayo/agosto.

Ovejero, A. (1996). "Psicología social de la educación". En J. L. Alvaro, A. Garrido y J. R. Torregrosa (Cord.), Psicología social aplicada. Madrid: McGraw Hill.

Peiró, J. M. y Prieto, F. (Dirs) (1996). Tratado de psicología del trabajo. Vol. I. La actividad laboral en su contexto. Madrid: Síntesis Psicología.

Pelechano, V. (Dir). (1989). "Análisis y modificación de conducta (tema monográfico)". Análisis y modificación de conducta. Vol. 15.

Perrenoud, Ph. (1990). La construcción del éxito y del fracaso escolar. Madrid. Morata.

Peterson, C. y Seligman, M. E. P. (1984). "Causal explanation as a risk factor for depression: Theory and evidence". Psychological Review, 91, 347-374.

Pozo, C. (1996). El fracaso académico en la Universidad: Sistema de evaluación e intervención preventiva. Tesis Doctoral (no publicada), Madrid: Universidad Autónoma de Madrid.

Pozo, C. y Hemández, J. M. (1997). "El fracaso académico universitario". En P. Apodaca y C. Lobato (Comps.), Calidad en la Universidad: Orientación y evaluación. Bilbao: Alertes.

Saldaña, R. (1986). Éxitos y fracasos en la universidad. En M. Latiesa (Comp.), Estudio longitudinal de una cohorte de alumnos de la Universidad Autónoma de Madrid. Análisis de la deserción universitaria (pp. 1-17). Madrid: Universidad Autónoma de Madrid.

Salvador, L. y Valcárcel, A. (1989). El rendimiento académico en la Universidad de Cantabria: Abandono y retraso en los estudios. Madrid: CIDE

Sánchez Miguel, E. (1995) Los textos expositivos, estrategias para mejorar su comprensión. Buenos Aires, Ed Santillana, 1995

Tyler, L. E. (1965). Psicología de las diferencias humanas. Madrid: Marova.

Vroom, V. H. (1976). "Can leaders learn to lead?”. Organizational Dynamics, (Winter), 17-28.

Zurita, N. I. C. y Leite, A. E. (1997). Perfil Sociomotivacional, Modalidad Institucional y Comportamiento Académico. Ponencia presentada en el Segundo Encuentro Nacional: La Universidad como objeto de investigación. Buenos. Aires.

Zurita, N. de (1996). "El Primer Año Universitario, variables Académicas y Extra-académicas asociadas al Abandono, la permanencia y el rendimiento Académico, Facultad de Humanidades". En Revista Nordeste $2^{\mathrm{a}}$ Época, Serie Investigaciones y Ensayos, 3 Ciencias de la Educación -UNNE- Resistencia. 


\section{ANEXO}

\section{Cuestionario}

El objetivo del cuestionario es conocer mejor la realidad y la problemática que rodea el proceso de Enseñanza - Aprendizaje de los universitarios.

Nota: el cuestionario es anónimo y los datos serán confidenciales. GRACIAS POR TU COLABORACIÓN.

\section{Datos sociográficos}

Edad:

Sexo:

$\square$ Hombre

Mujer

Estado civil:

$\square$ Soltero

$\square$ Casado

$\square$ Otras

Procedencia: $\quad \square$ Huelva y provincia Otras provincias:
Ámbito rural
Ámbito urbano

Diplomatura/licenciatura de:

Especialidad de:

Año de finalización de los estudios de bachillerato:

Residencia actual: Domicilio paterno

Domicilio propio

$\begin{array}{llll} & \square \text { Piso de estudiante } & \square \text { Pensión } & \square \text { Colegio mayor/residencia } \\ \text { Situación laboral: } & \square \text { Desempleado } & \square \text { Activo } & \\ \text { Tipo de empleo: } & \square \text { Eventual } & \square \text { Fijo } & \\ \text { Jornada laboral: } & \square \text { Mañana } & \square \text { Tarde } & \square \text { Turnos } \quad \square \text { Mañana y tarde } \\ \text { Estudias con beca: } & \square \text { Sí } & \square \text { No } & \end{array}$

1. ¿Cuántos libros que no son obligatorios de tu carrera y que no te aporten nota lees en un cuatrimestre?

Menos de 2 libros $\square$ Entre 2 y 4 libros $\square$ Más de 4 libros

2. ¿Cuántos libros obligatorios lees cuatrimestralmente?

$\square$ menos de 5 libros $\square$ entre 5 y 10 libros $\square$ más de 10 libros

3. ¿Complementas la información de las asignaturas con otras lecturas?
$\square$ Sí
$\square$ No

4. ¿Qué genero literario te gusta?

$\square$ Ensayos $\quad \square$ Novelas $\square$ Poesía $\square$ Otros

5. ¿Qué tipo de revistas lees? 
6. ¿Crees que tus padres fomentan la lectura en casa?

$\square$ Sí $\quad \square$ No

7. ¿Qué tiempo dedicas al estudio semanalmente?

$\square 10$ horas

$\square$ Entre 10 y 20 horas

Más de 20 horas

8. Habitualmente estudio:

Un poco todos los días

Sólo los fines de semana

Sólo para los exámenes

9. Señala la frecuencia de uso de las siguientes herramientas.

\begin{tabular}{|l|l|l|l|}
\cline { 2 - 4 } \multicolumn{1}{c|}{} & Generalmente & A veces & No lo uso \\
\hline Apuntes de clase & & & \\
\hline Asistencia a clase (cuando no es obligatorio) & & & \\
\hline Material fotocopiado & & & \\
\hline Libros de texto & & & \\
\hline Otros libros de consulta & & & \\
\hline Medios audiovisuales & & & \\
\hline Medios informaticos & & & \\
\hline
\end{tabular}

10. Señala los procedimientos que utilizas:

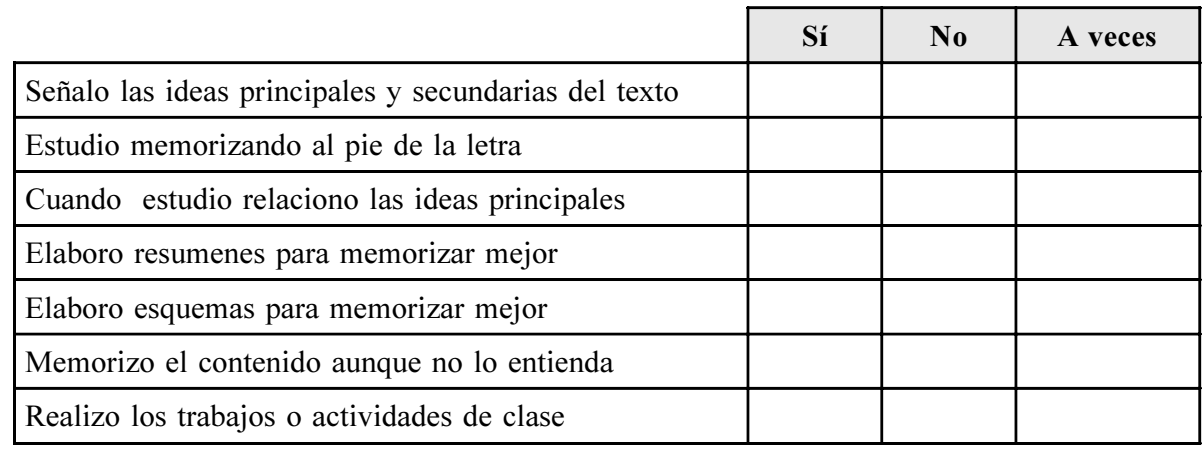

11. ¿Qué tipo de exámenes prefieres?

¿Por qué?

De desarrollo

Tipo test

Combinado

12. ¿En qué tipo de exámenes consigues mayores calificaciones?
$\square$ De desarrollo
$\square$ Tipo test
Combinado

¿A qué crees que es debido? 
13. ¿En el examen tipo test qué dificultades encuentras?

\begin{tabular}{|l|l|l|l|}
\cline { 2 - 4 } \multicolumn{1}{c|}{} & \multicolumn{1}{c|}{ Nada } & Algo & Mucho \\
\hline Preguntas largas & & & \\
\hline Preguntas confusas & & & \\
\hline No se entendie el vocabulario & & & \\
\hline La pregunta es de aplicación práctica & & & \\
\hline
\end{tabular}

14. ¿En el examen de desarrollo qué dificultades encuentras?

\begin{tabular}{|l|l|l|l|}
\cline { 2 - 4 } \multicolumn{1}{c|}{} & Nada & Algo & Mucho \\
\hline Falta de planificación temporal & & & \\
\hline No entender lo que preguntan & & & \\
\hline El modo de estudiar la asignatura & & & \\
\hline La pregunta es de aplicación práctica & & & \\
\hline Dificultad para estructurar la repuestas & & & \\
\hline
\end{tabular}

15. ¿Resulta fácil responder por escrito con coherencia y lógica?
$\square$ Sí
$\square$ No

16. ¿Eres capaz de organizar los conocimientos que tienes de la asignatura para expresarlo en un examen a desarrollar?
$\square$ Sí
$\square$ No

Si contestas no, ¿a qué crees que es debido?

17. Problemas y obstáculos que pueden interferir en tus estudios.

\begin{tabular}{|l|l|l|l|}
\cline { 2 - 4 } \multicolumn{1}{c|}{} & \multicolumn{1}{c|}{ Nada } & Algo & Mucho \\
\hline Falta de esfuerzo & & & \\
\hline Falta de interés & & & \\
\hline Falta de capacidad & & & \\
\hline Los profesores & & & \\
\hline Problemas afectivos & & & \\
\hline Ansiedad & & & \\
\hline Problemas familiares & & & \\
\hline "Salidas nocturnas" & & & \\
\hline Cantidad de trabajos & & & \\
\hline Deficiencias en tu formación previa & & & \\
\hline
\end{tabular}

Fecha de recepción: 30-10-03

Fecha de revisión: 17-03-05

Fecha de aceptación: 20-04-05 